\title{
Tunable magnetic interaction at the atomic scale in oxide heterostructures
}

\author{
J. W. Seo ${ }^{1,2}$, W. Prellier ${ }^{3}$, P. Padhan ${ }^{3,4}$, P. Boullay ${ }^{3}$, J.-Y. Kim ${ }^{5}$, Hangil Lee ${ }^{5,6}$, C. D. \\ Batista $^{7}$, I. Martin ${ }^{7}$, Elbert. E. M. Chia ${ }^{2}$, T. Wu ${ }^{2}$, B. -G. Cho ${ }^{8}$ and C. Panagopoulos ${ }^{1,2,9}$ \\ ${ }^{1}$ Cavendish Laboratory, University of Cambridge, Cambridge CB3 OHE, UK \\ ${ }^{2}$ Division of Physics and Applied Physics, Nanyang Technological University, 637371 Singapore \\ ${ }^{3}$ Laboratoire CRISMAT, CNRS UMR 6508, ENSICAEN, 14050 Caen Cedex, France \\ ${ }^{4}$ Department of Physics, Indian Institute of Technology Madras, Chennai - 600036, India \\ ${ }^{5}$ Pohang Accelerator Laboratory, Pohang 790-784, South Korea \\ ${ }^{6}$ Department of Chemistry, Sookmyung Women's University, Seoul 140-742, South Korea \\ ${ }^{7}$ Theoretical Division, Los Alamos National Laboratory, Los Alamos, New Mexico 87545, USA \\ ${ }^{8}$ Department of Physics, Pohang University of Science and Technology (POSTECH), Pohang 790-784, South Korea and \\ ${ }^{9}$ Department of Physics, University of Crete and FORTH, 71003 Heraklion, Greece
}

\begin{abstract}
We report on a systematic study of a number of structurally identical but chemically distinct transition metal oxides in order to determine how the material-specific properties such as the composition and the strain affect the properties at the interface of heterostructures. Our study considers a series of structures containing two layers of ferromagnetic $\mathrm{SrRuO}_{3}$, with antiferromagnetic insulating manganites sandwiched in between. The results demonstrate how to control the strength and relative orientation of interfacial ferromagnetism in correlated electron materials by means of valence state variation and substrate-induced strain, respectively.
\end{abstract}

The Giant Magneto-Resistance effect in metallic magnetic multilayer systems forms the basis of highly successful magnetic sensing and storage technology [1, 2]. The active search for new materials that would allow for ever higher sensitivity and controllability is under way. Transition metal oxides (TMO) are particularly attractive, since there is a plethora of isostructural materials with a wide variety of magnetic and electronic properties, which can be seamlessly built into complex heterostructures 3 -10]. In heterostructures composed of different TMO, the disruption introduced even by an ideal interface, can drastically upset the delicate balance of the competing interactions among electronic spins, charges and orbitals, leading to a range of exotic phenomena, including interfacial conduction, magnetism, and superconductivity [3-10]. Among TMO, Mn-based perovskites $\mathrm{AMnO}_{3}$ (manganites) are one of the best studied classes of materials that exhibit particularly rich set of behaviors tunable by composition, pressure and temperature [11]. It has been recently demonstrated that when put into contact with ferromagnetic (FM) $\mathrm{SrRuO}_{3}$, the interface layer of antiferromagnetic (AF) manganite becomes FM [12]. In this work we report a strategy and evidence for manipulating the magnetic properties at the atomic level in digitally synthesized nano-heterostructures. We engineer interfacial FM in correlated electron materials by means of valence state variation and substrate-induced strain.

Trilayers, $\left[\mathrm{SrRuO}_{3} /\right.$ manganite $\left./ \mathrm{SrRuO}_{3}\right]$, were grown simultaneously on (001)-oriented $\mathrm{SrTiO}_{3}$ and $\mathrm{LaAlO}_{3}$ substrates using the multitarget pulsed laser deposition technique with energy density of $3 \mathrm{~J} / \mathrm{cm}^{2}$. The conditions for optimizing the deposition can be found elsewhere [13]. In this study, we chose three types of AF insulating manganites: $\mathrm{CaMnO}_{3}$ with
$\mathrm{Mn}^{4+}$ (orbital state $t_{2 g}^{3}$ ), $\operatorname{Pr}_{0.5} \mathrm{Ca}_{0.5} \mathrm{MnO}_{3}$ with $\mathrm{Mn}^{3+/ 4+}$ and $\mathrm{PrMnO}_{3}$ with $\mathrm{Mn}^{3+}\left(t_{2 g}^{3} e_{g}^{1}\right)$. The schematic drawing and high resolution electron microscopy (HREM) image of the trilayers $\left[18\right.$ unit cell (u.c.) $\mathrm{SrRuO}_{3} / 4$ u.c. $\mathrm{Pr}_{0.5} \mathrm{Ca}_{0.5} \mathrm{MnO}_{3} / 18$ u.c. $\mathrm{SrRuO}_{3}$ ] deposited on $\mathrm{SrTiO}_{3}$ is depicted in Fig. 1(a). HREM was performed on crosssection specimens prepared by mechanical polishing followed by ion-milling. The typical HREM image, viewed along the [001] direction (Fig. 1(a)) reveals the epitaxial growth of the trilayers onto the $\mathrm{SrTiO}_{3}$ substrate. Structural characterization of the trilayers with $\mathrm{CaMnO}_{3}$ manganite layer was performed using X-ray diffraction (XRD) 14] and XRD mapping with photon energy of $11 \mathrm{KeV}$ as shown in Fig. 1(b) and (c). The XRD maps of a reciprocal space in the vicinity of $(h k l)=(103)$ reflection were performed at room temperature at beamline $10 \mathrm{~A}$ of the Pohang Accelerator Laboratory in Korea using four-circle diffractometers. The typical twin structure of the $\mathrm{LaAlO}_{3}$ substrate gives rise to the broad peak at (103) 15]. The red and blue colors indicate high and low scattering intensity, respectively. The maps show the inplane lattices of the substrates and the films match each other without lattice relaxations. The ratio of in-plane to out-of-plane lattice parameters $(a / c)$ estimated by the maps is 0.94 (strong compressive strain) in the case of films grown on $\mathrm{LaAlO}_{3}$ (Fig. 1(b)), whereas $a / c \sim 0.98$ (weak compressive strain) in the case of $\mathrm{SrTiO}_{3}$ (Fig. $1(\mathrm{c}))$. The substrate $c$-axis parameters obtained from $\theta$ $2 \theta$ scans (the middle panels of (b) and (c)) at the $h=1$ reflections (guided by the black vertical line on the maps) are $3.789 \AA$ and $3.906 \AA$, respectively. An earlier study reports that all the heterostructures we study here have the same Curie temperature $\left(\mathrm{T}_{C}\right)$ i.e., approximately $150 \mathrm{~K}$, close to the $\mathrm{T}_{C}$ of the $\mathrm{FM} \mathrm{SrRuO}_{3}$ film and independent of the substrates and AF spacers [14]. This small vari- 


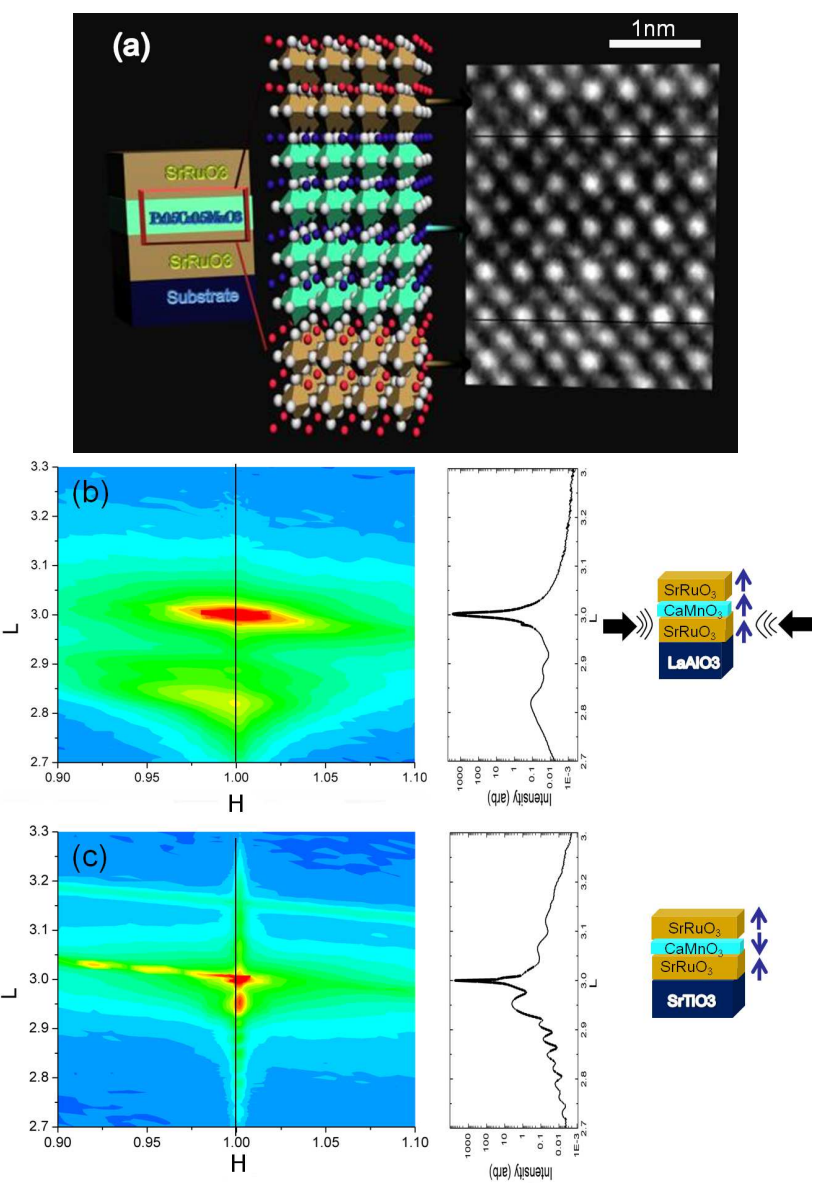

FIG. 1: (a) Schematic structure (left and middle panels) of the trilayer $\left[18\right.$ u.c $\mathrm{SrRuO}_{3} / 4$ u.c $\mathrm{Pr}_{0.5} \mathrm{Ca}_{0.5} \mathrm{MnO}_{3} / 18$ u.c $\mathrm{SrRuO}_{3}$ ] on $\mathrm{SrTiO}_{3}$. The high resolution electron microscope (HREM) image (right panel) shows the epitaxial growth of the sample on $\mathrm{SrTiO}_{3}$. Reciprocal space maps (left panels) of the trilayer $\left[\mathrm{SrRuO}_{3} / \mathrm{CaMnO}_{3} / \mathrm{SrRuO}_{3}\right]$ grown on (b) $\mathrm{LaAlO}_{3}$ and (c) $\mathrm{SrTiO}_{3}$ near $(h k l)=(103)$. The schematic at the right hand side of panel (b) depicts the switching/reversal of the spin direction along the $c$ axis due to the effect of strong compressive strain arising from the $\mathrm{LaAlO}_{3}$ substrate.

ation in $\mathrm{T}_{C}$ indicates the absence of intermixing at the interfaces. The coercive fields measured at $80 \mathrm{~K}$ in a magnetic field applied perpendicular to the films (parallel to the easy axis) are $0.2 \mathrm{~T}$ and $0.5 \mathrm{~T}$ for the films grown on $\mathrm{SrTiO}_{3}$ and $\mathrm{LaAlO}_{3}$, respectively. The saturated magnetic moments vary from 1 to $1.3 \mu_{B}$ / unit cell depending on the manganite layers.

We studied the magnetic profile of the manganite layers in the devices using atom-selective X-ray magnetic circular dichroism (XMCD) at the absorption edge of Mn $\mathrm{L}_{2,3}$. XMCD measurements were performed at beamline $2 \mathrm{~A}$ of the Pohang Accelerator Laboratory in Korea. All XMCD data were obtained after zero-field cooling to $80 \mathrm{~K}$. We measured the total yield signal with an energy resolution of $200 \mathrm{meV}$ in a magnetic field of $0.9 \mathrm{~T}$, perpendicular to the plane of the film. Our approach allows
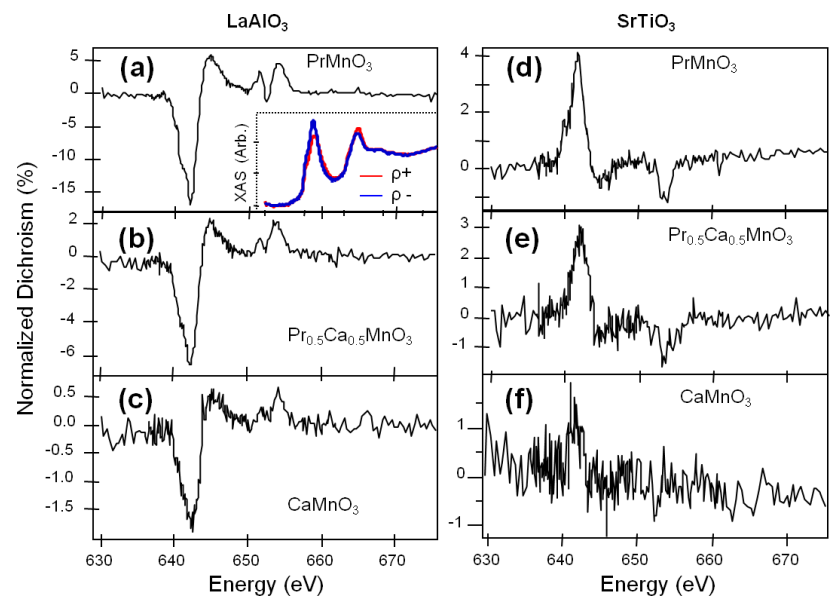

FIG. 2: $\mathrm{Mn} \mathrm{L}_{2,3}$ edge XMCD (black: $\rho^{+}-\rho^{-}$) spectra of the manganite layer in the trilayer $\left[\mathrm{SrRuO}_{3} /\right.$ manganite $\left./ \mathrm{SrRuO}_{3}\right]$. Spectra are taken at $80 \mathrm{~K}$ in a magnetic field of $0.9 \mathrm{~T}$ applied perpendicular to the plane of the film. (a)-(c) and (d)-(f) depict spectra for the samples deposited on $\mathrm{LaAlO}_{3}$ and $\mathrm{SrTiO}_{3}$, respectively. Inset in panel (a): $\mathrm{Mn}_{2,3}$ XAS (red: $\rho^{+}$, blue: $\left.\rho^{-}\right)$spectra.

for investigation of a buried layer without spurious signals from neighbouring layers or the substrate. The measurement was performed by saturating the magnetization of $\mathrm{SrRuO}_{3}$ layers in the $c$-direction by a magnetic field in excess of the coercive field. Figure 2 depicts the $\mathrm{Mn}$ dichroism spectra $\left(\rho^{+}-\rho^{-}\right)$, i.e., the difference between Xray absorption spectroscopy (XAS) data taken with the helicity parallel and antiparallel to the applied magnetic field. The spectra are normalized to the intensity of $\rho^{+}+$ $\rho^{-}$at the $\mathrm{L}_{3}$ peak. Notably, Mn dichroism for the samples deposited on $\mathrm{LaAlO}_{3}$ (Fig. 2(a)-(c)) has the opposite sign to those grown on $\mathrm{SrTiO}_{3}$ (Fig. 2(d)-(f)), implying that the substrate determines the sign of the magnetic coupling between $\mathrm{Mn}$ and $\mathrm{Ru}$ across the interface. The schematic drawings in Fig. 1(b) and (c) (right panels) show that the spin direction depends on the substrates the films are grown onto. The large arrows in Fig. 1(b) (right panel) indicate the strong compressive strain is due to the $\mathrm{LaAlO}_{3}$ substrate.

Table 1 summarizes the values of the magnetization for the three different manganite layers as deduced from the normalized XMCD, $\left(\rho^{+}-\rho^{-}\right) /\left(\rho^{+}+\rho^{-}\right)(\%)$. In the case of $\mathrm{PrMnO}_{3}$ grown on the $\mathrm{LaAlO}_{3}$ substrate, the value of $17 \%$ has been estimated to correspond approximately to $1.7 \mu_{B} / \mathrm{Mn}$ determined by the sum rule [16]. The negative sign for the samples grown on $\mathrm{SrTiO}_{3}$ indicates that the spins of the manganite layer point antiparallel to the applied magnetic field. Notably, the magnetization of the manganite layers deposited on $\mathrm{SrTiO}_{3}$ is weaker compared to the heterostructures deposited on $\mathrm{LaAlO}_{3}$. These results indicate that the substrate influences the strength and the direction of the induced magnetization in the manganite layer. Our findings demon- 
TABLE I: Magnetization of three types of spacer manganite layers $\left(\mathrm{CaMnO}_{3}, \mathrm{Pr}_{0.5} \mathrm{Ca}_{0.5} \mathrm{MnO}_{3}\right.$, and $\left.\mathrm{PrMnO}_{3}\right)$ deposited on two different substrates, namely $\mathrm{LaAlO}_{3}$ and $\mathrm{SrTiO}_{3}$. The values of the magnetization are deduced by normalized XMCD signals defined as $\left(\rho^{+}-\rho^{-}\right) /\left(\rho^{+}+\rho^{-}\right)$.

\begin{tabular}{|l|c|c|r|}
\hline & $\mathrm{CaMnO}_{3}$ & $\mathrm{Pr}_{0.5} \mathrm{Ca}_{0.5} \mathrm{MnO}_{3}$ & $\mathrm{PrMnO}_{3}$ \\
\hline $\mathrm{LaAlO}_{3}$ & $2 \%$ & $6.5 \%$ & $17 \%$ \\
\hline $\mathrm{SrTiO}_{3}$ & $-1.5 \%$ & $-3 \%$ & $-4 \%$ \\
\hline
\end{tabular}

strate that one may be able to prepare tailor-made devices with the desired direction and magnitude of the moment in the nanometer-thin FM interface, by depositing the multilayer on a pre-specified substrate. Notably, the observed magnitude of the magnetization follows the order: $\mathrm{PrMnO}_{3}>\mathrm{Pr}_{0.5} \mathrm{Ca}_{0.5} \mathrm{MnO}_{3}>\mathrm{CaMnO}_{3}$, particularly evident for the case of $\mathrm{LaAlO}_{3}$ substrate.

The sign and strength of the inter-ion magnetic exchange interaction is commonly analyzed within the Goodenough-Kanamori (GK) rules [17]. Depending on the orbital occupancy and the presence or absence of an overlap between the orbitals belonging to the two ions, the exchange can be either FM or AF. For instance, for half-filled orbitals with non-zero overlap the exchange is $\mathrm{AF}$ [18]. Let us examine what would be the consequences of the GK rules in our system. $\mathrm{Ru}^{4+}$ has four electrons in the $t_{2 g}$ orbitals. Three of them are ferromagnetically aligned by the onsite Hund's interaction, while the fourth is antialigned with the first three, yielding a state with spin $S=1$ (the crystal field splitting between $e_{g}$ and $t_{2 g}$ orbitals of Ru exceeds the onsite FM Hund's coupling, which leaves the $\mathrm{Ru} e_{g}$ orbital unoccupied).

In $\mathrm{CaMnO}_{3}$, Mn state is $t_{2 g}^{3}(S=3 / 2)$, which corresponds to half-filled $t_{2 g}$ band. The Mn atom is connected with $\mathrm{Ru}$ via apical oxygen, which leads to one-to-one hybridization between the respective orbitals, e.g. $\mathrm{Ru} t_{2 g}$ $x z(y z)$ with Mn $t_{2 g} x z(y z)$ and $\mathrm{Ru} e_{g} 3 z^{2}-r^{2}$ with $\mathrm{Mn}$ $e_{g} 3 z^{2}-r^{2}(z \|$ interface normal). Note that by symmetry, there is no oxygen-mediated hybridization between the $x y$ orbitals of $\mathrm{Ru}$ and $\mathrm{Mn}$ (we neglect the possible but small symmetry-breaking effects of lattice distortions), while the direct overlap between the orbitals is very weak. The same is true for the $x^{2}-y^{2} e_{g}$ orbitals. If $\mathrm{Ru}$ were in $t_{2 g}^{3}$ fully polarized state, the GK rules would imply that the coupling between $\mathrm{Ru}$ and $\mathrm{Mn}$ is $\mathrm{AF}$. However, experimentally we find that on $\mathrm{LaAlO}_{3}$ substrate the coupling is FM. Clearly the FM coupling can only be caused by the fourth - the minority - electron of Ru. In the limit of small hybridization between $\mathrm{Ru}$ and $\mathrm{Mn}$ ions, the sign and magnitude of the magnetic coupling can be understood by means of perturbation theory in hopping $t$. The virtual electron hopping processes transfer electrons between low and high-energy ionic sates (the energy differ- ence must be much bigger than $t$ for the perturbation theory to be valid). FM coupling induced by hopping of the minority electron can become dominant if the energy barrier associated with the transfer of this electron between FM aligned $\mathrm{Ru}$ to $\mathrm{Mn}$ ions is considerably smaller than for the same process for $\mathrm{AF}$ alignment of $\mathrm{Ru}$ and $\mathrm{Mn}$. We show in the following that there is indeed a significant range of realistic parameters where FM coupling dominates. Whether or not the minority electron contributes to the magnetic coupling between $\mathrm{Ru}$ and $\mathrm{Mn}$ is determined by the strain, which controls the relative energies of the $t_{2 g}$ orbitals of Ru. For strong compressive inplane strain, such as the one induced by the $\mathrm{LaAlO}_{3}$ substrate, the $x z, y z$ orbitals are lower than the $x y$ orbital (similarly, $e_{g} 3 z^{2}-r^{2}$ is lower than $x^{2}-y^{2}$ ). These are the orbitals that hybridize across the interface, and therefore when the minority-spin electron of Ru occupies either one of them, it can mediate FM coupling between $\mathrm{Ru}$ and $\mathrm{Mn}$. For the tensile strain, however, the minority electron of $\mathrm{Ru}$ occupies the $x y$ orbital and does not hybridize with $\mathrm{Mn}$, which leaves only the AF channel open.

To test this qualitative argument, we performed exact diagonalization studies of the $\mathrm{Mn}^{4+}-\mathrm{Ru}^{4+}$ complex including all $d$ orbitals for various values of effective $\mathrm{Ru}-$ Mn hopping integral $t$ and strain-induced crystal field splitting, $\delta=\varepsilon_{x y}-\varepsilon_{x z, y z}$ (Fig. 3). The hopping integral is taken to be zero between the $d$ orbitals for which by symmetry there is no oxygen-mediated hybridization ( $x y$ case). The spin state of isolated $\mathrm{Mn}$ ion is $S_{\mathrm{Mn}}=3 / 2$ and of isolated $\mathrm{Ru}$ ion is $S_{\mathrm{Ru}}=1$. The Hamiltonian is

$$
\begin{aligned}
H_{0} & =\sum_{j, \alpha} \varepsilon_{j} n_{j \alpha}+\sum_{j, \alpha} U_{j} n_{j \alpha \uparrow} n_{j \alpha \downarrow}+\sum_{j, \alpha \neq \alpha^{\prime}} \frac{U_{j}-J_{j}}{2} n_{j \alpha} n_{j \alpha^{\prime}} \\
& -\sum_{j, \alpha \neq \alpha^{\prime}} \frac{J_{j}}{2}\left[\mathbf{S}_{j \alpha} \cdot \mathbf{S}_{j \alpha^{\prime}}+\frac{1}{4} n_{j \alpha} n_{j \alpha^{\prime}}-d_{j \alpha \uparrow}^{\dagger} d_{j \alpha \downarrow}^{\dagger} d_{j \alpha^{\prime} \downarrow} d_{j \alpha^{\prime} \uparrow}\right] \\
& +\delta \sum_{j}\left(n_{j x z}+n_{j y z}\right)+\sum_{\alpha, \alpha^{\prime}, \sigma} t_{\alpha \alpha^{\prime}}\left(d_{1 \alpha \sigma}^{\dagger} d_{2 \alpha^{\prime} \sigma}+\text { H.c. }\right)
\end{aligned}
$$

where $j=\mathrm{Mn}^{4+}, \mathrm{Ru}^{4+}, U_{j}$ are the Coulomb repulsions and $J_{j}$ are the Hund's coupling constants. The orbital label $\alpha$ takes the values $\alpha=\{x y, x z, y z\}$, while $\sigma=\uparrow, \downarrow$ is the spin label. Finally, $n_{j \alpha \sigma}=d_{j \alpha \sigma}^{\dagger} d_{j \alpha \sigma}$ and $n_{j \alpha}=\sum_{\sigma} n_{j \alpha \sigma}$. The hopping matrix is diagonal, $t_{\alpha, \alpha^{\prime}}=t_{\alpha, \alpha} \delta_{\alpha, \alpha^{\prime}}$, with $t_{x y, x y}=0, t_{x z, x z}=t_{y z, y z}=t$. We use parameters 19] $J_{\mathrm{Ru}}=J_{\mathrm{Mn}}=1.5 \mathrm{eV}, U_{\mathrm{Mn}}=5$ $\mathrm{eV}, U_{\mathrm{Ru}}=4 \mathrm{eV}, \varepsilon_{\mathrm{Mn}}-\varepsilon_{\mathrm{Ru}}=-2 \mathrm{eV}$. We find that for $\delta \gtrsim \delta_{c}(t)$ (strong compressive strain) where $\delta_{c}(t)$ is a critical value of $\delta$, the sates with high total spin $\left(S_{t o t}=5 / 2\right.$ and $S_{t o t}=3 / 2$ ), are stabilized, which corresponds to FM coupling between $\mathrm{Mn}$ and $\mathrm{Ru}$. The critical value of $\delta$ for the transition between $S_{t o t}=1 / 2$ and $S_{t o t}=3 / 2$ depends on the value of $t$ (see Fig.3 (a)). We point out that the state with $S_{t o t}=3 / 2$ that appears at larger values of hopping $t$ is beyond the ionic picture. On the other hand, for $\delta<\delta_{c}(t)$ (tensile or weak compressive strain) 
(a)

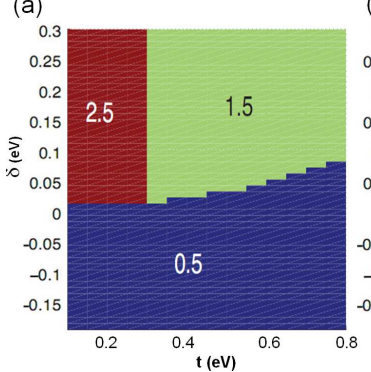

(b)

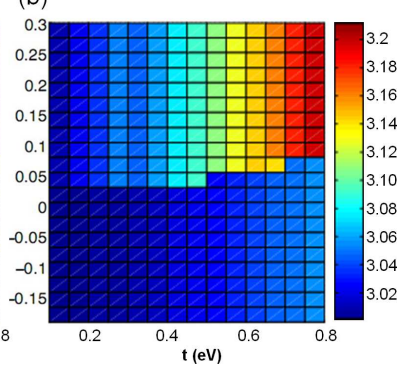

FIG. 3: (a) The total spin of coupled $\mathrm{Mn}^{4+}(S=3 / 2)$ and $\mathrm{Ru}^{4+}(S=1)$ as a function of inter-ion $t_{2 g}$ hybridization $t$ and the crystal field splitting $\delta=\varepsilon_{x y}-\varepsilon_{x z, y z}$. (b) The charge of the $\mathrm{Mn}$ ion. The transitions between different total spin states are accompanied by the change in the charge of the ions.

the $\mathrm{Ru}$ and $\mathrm{Mn}$ ions are anti-aligned into the state with lowest total spin $\left(S_{t o t}=1 / 2\right)$. This is in agreement with the above qualitative discussion.

In $\mathrm{PrMnO}_{3}$, the nominal valence of $\mathrm{Mn}$ is $3+$, i.e., there is one extra electron in the $e_{g}$ orbital of Mn. Due to the Hund coupling, the spin of this electron is aligned with the spins of the other $\left(t_{2 g}\right)$ electrons of $\mathrm{Mn}$. In the case of strong compressive inplane strain $\left(\mathrm{LaAlO}_{3}\right.$ substrate) the extra $e_{g}$ electron occupies the $3 z^{2}-r^{2}$ orbital of $\mathrm{Mn}$, which is well hybridized with the corresponding empty orbital of $\mathrm{Ru}$, and therefore according to the GK rules favors FM alignment of $\mathrm{Mn}$ and $\mathrm{Ru}$ spins. This contribution adds to the FM coupling that is already present in the case of $\mathrm{CaMnO}_{3} / \mathrm{SrRuO}_{3}$ interface. In addition, the magnetic moment is bigger for the $\mathrm{Mn}^{3+}$ ion $\left(S_{\mathrm{Mn}}=2\right)$. Both effects lead to the relatively enhanced value of the induced FM moment in $\operatorname{Pr}_{0.5} \mathrm{Ca}_{0.5} \mathrm{MnO}_{3}$ and $\mathrm{PrMnO}_{3}$, in agreement with the progression that we observe experimentally (Table 1). On the other hand, for tensile or weak compressive inplane strain $\left(\mathrm{SrTiO}_{3}\right.$ substrate), the extra electron on Mn occupies the $e_{g} x^{2}-y^{2}$ orbital, which by symmetry cannot hybridize with the apical oxygen orbitals and thus does not have significant overlap with the $\mathrm{Ru}$ d-orbitals across the interface. Thus we conclude that for tensile or weak compressive inplane strain, just as in the case of $\mathrm{CaMnO}_{3}$, in $\mathrm{Pr}_{0.5} \mathrm{Ca}_{0.5} \mathrm{MnO}_{3}$ and $\mathrm{PrMnO}_{3}$, there is no virtual hopping process that would favor FM alignment of $\mathrm{Mn}$ and $\mathrm{Ru}$ ions, and the result is a (relatively weak) AF coupling between Mn and Ru ions. This is in complete qualitative agreement with our experimental observations.

In summary, we report direct evidence for tunable FM behavior at the atomic scale in strongly correlated oxide heterostructures. We find that the orientation and strength of the induced interfacial magnetism can be very sensitive to strain. By selecting appropriate substrate one may now design complex magnetic heterostructures with the desired relative arrangement of the magnetic elements, of potential utility to electronics and spintronics, including magnetic memory and sensing. Moreover, the strain can be also induced by means of externally applied force, which can thus cause mechanically-induced magnetic reorientation. For example, by using a piezoelectric substrate such as PNM-PT $\left(\mathrm{Pb}\left(\mathrm{Mg}_{1 / 3} \mathrm{Nb}_{2 / 3}\right) \mathrm{O}_{3^{-}}\right.$ $\left.\mathrm{PbTiO}_{3}\right)$ [20] one would be able to control the orientation and strength of the magnetization by tuning the lattice parameters by means of an applied electric field.

This work was supported by MEXT-CT-2006-039047, EURYI, KRF-2005-215-C00040, LAFICS, STAR, CEFIPRA/IFPCAR, National Research Foundation of Singapore, and the Merlion project (n2.04.07). The authors acknowledge $\mathrm{H}$. Hwang for helpful comments on the Goodenough-Kanamori rules, and A. Pautrat and Y. Tokura for useful discussions.

[1] G. Binasch, P. Grünberg, F. Saurenbach, W. Zinn, Phys. Rev. B 39, 4828 (1989).

[2] M. N. Baibich, et al., Phys Rev. Lett. 61, 2472 (1988).

[3] Y. Tokura, N. Nagaosa, Science 288, 462(2000).

[4] A. Ohtomo, H. Y. Hwang, Nature 427, 423(2004).

[5] A. Brinkman, et al., Nature Materials 6, 493 (2007).

[6] A. Gozar, et al., Nature 455, 782 (2008).

[7] S. Thiel, G. Hammerl, A. Schmehl, C. W. Schneider, J. Mannhart, Science 313, 1942 (2006).

[8] J. Heber, Nature 459, 28 (2009).

[9] G. Logvenov, A. Gozar, I. Bozovic, Science 326, 699 (2009).

[10] J. Mannhart, D. G. Schlom, Science 327, 1607 (2010).

[11] Y. Tokura, Colossal Magnetoresistive Oxides Advances in Condensed Matter Science, vol. 2. (Gordon and Breach Science Publishers, 2000).

[12] Y. Choi, et al., Appl. Phys. Lett. 91, 022503 (2007).

[13] P. Padhan, W. Prellier, B. Mercey, Phys. Rev. B 70, 184419 (2004).

[14] P. Padhan, W. Prellier, Phys. Rev. B 76, 024427 (2007).

[15] C. Aruta, et al., J. Appl. Phys. 100, 023910 (2006).

[16] C. T. Chen, et al., Phys. Rev. Lett. 75, 152 (1995).

[17] J. Kanamori, J. Prog. Theoret. Phys. 17, 177 (1957); J. B. Goodenough, J. Phys. Chem. Solids 6, 287 (1958).

[18] P. W. Anderson, Phys. Rev. 79, 350 (1950).

[19] J. M. D. Coey, M. Viret, S. Von. Molnar, Adv. in Phys. 48, 167 (1999)

[20] O. Bilani-Zeneli, et al., J. Appl. Phys. 104, 054108 (2008). 